\title{
SU ALCUNI USI TEMPO-ASPETTUALI DEI PARADIGMI VER- BALI ITALIANI TRAPASSATO PROSSIMO E IMPERFETTO
}

\section{Presentazione della problematica}

Nel presente contributo mi propongo di discutere certe scelte del TRAPASSATO PROSSIMO (TP) e dell'IMPERFETTO (IM), avanzando suggerimenti sui principi che le regolano. Ero partita da alcune ricorrenti soluzioni testuali italiane che, con la propria veste linguistica, in uno straniero di solito provocano delle perplessità, ad es.:

Situazioni nell'attualità

(1.a)

(uno dei visitatori, a un'ora tarda, alla porta d'ingresso di una coppia, già in pigiama)

- Scommetto che AVEVATE RINUNCIATO a vederci stasera. (LSE)

(1.b)

(davanti a un mucchio di cocci, la madre al bambino)

- Te l'AVEVO DETTO di non toccarlo! (LSE)

(1.c)

(cade un oggetto e si rompe)

- Il vaso di cristallo! L'unica cosa di valore che ERA RIMASTA in questa casa! (RAI 1, 24.10.98)

(1.d)

(dialogo relativo alla soluzione di un mistero)

"Ma non potrebbero essere le anime dei bibliotecari trapassati che fanno queste magie?"

Nicola ristette perplesso e inquieto: "A questo non AVEVO PENSATO. Può darsi. (...)." (Eco 98)

(1.e)

(un impiegato, mentre il capo viene portato nell'ufficio sul letto ospedaliero)

Non l'ASPETTAVAMO di ritorno così presto, signor direttore! (LSE 3577-8)

Situazione nell'attualità allargata

(2)

(tra due amici)

Il mio psicanalista mi fa tirar fuori cose che non RICORDAVO affatto... e poi mi dà un conto che non dimenticherò mai. (LSE 3678-42) 
Situazione nell'avvenire

(3)

(un troglodita a un altro che ha appena disegnato alla maniera "di oggi" un volto di donna)

Distruggilo! Vuoi forse che i posteri pensino che non ERAVAMO capaci di disegnare?

(LSE 3522-29)

Situazione nell'extratemporalità

(4)

La donna fatica dieci anni per cambiare le abitudini del marito e poi si lamenta che non è più l'uomo che AVEVA SPOSATO. (LSE 3655-48)

Commento espositivo

(5)

(...) Chiarito questo torniamo alla storia dei parlanti italiani.

D A L ' 400 A L ' 700

ERAVAMO RIMASTI al ' 400 , con l'Italia divisa in tanti piccoli stati. In questo periodo il popolo parlava in ogni regione il dialetto del posto. (CD 54-55)

Azioni passate successe una dopo l'altra

(6.a)

I giocatori granata si gettarono all'attacco; e in un batter d'occhio AVEVANO CAPOVOLTO il risultato: da 0-2 a 3-2 (BER 1986a:459; Kor 2002:206))

(6.b)

('inizio di un compito in classe di una bambina)

“Mio zio Ernesto AVEVA COMPRATO un'auto. Alla prima uscita, però, andò a sbattere contro un albero. E, con questo, penso di aver scritto quasi trenta parole. (...) (LSE 3659-12)

Situazione nel passato espressa al procedimento storico

(7)

Cresciuto nella casa di Gandales di Scozia, che pietosamente l'HA STRAPPATO alle acque e lo HA ADOTTATO come figlio, Amadigi viene più tardi condotto da re Langrines alla propria corte. (...) A sua volta Amadigi, incantenato da forze occulte e segregato da tutti, è liberato da Oriana, che gli si concede spontaneamente per virtù d'amore (1.35). La bellezza a cui Amadigi ASPIRAVA e per la quale AVEVA tanto COMBATTUTO e tanto SOFFERTO, gli è data in dono come premio della sua fedeltà. (BOM 114-5, Amadigi di Gaula)

Nel caso di (1)-(7) si tratta di testi o segmenti testuali - e di situazioni comunicative - diversi fra di loro; ciò che li accomuna è l'opacità del motivo della scelta della forma verbale: perché proprio il Trapassato? Del resto, nemmeno la scelta dell'Imperfetto, specialmente in (3), si può semplicemente spiegare con "un'azione che durava nel passato" o con "un uso modale". 


\section{Relazioni tempo-aspettuali nel mondo testuale e paradigmi verbali}

Nell'affrontare il tema specifico $\mathrm{mi}$ sono servita dell'apparato teorico elaborato negli anni sulla base di analisi sistematiche di caratteristiche retoriche e, in particolare, della distribuzione dei paradigmi verbali italiani in testi di tipo diverso, cercando di cogliervi delle regolarità e ricorrenze sufficientemente convincenti. La risultante teoria tempo-aspettuale ${ }^{1}$ potrebbe essere riassunta così (cfr. Miklič 1997):

Di fronte a un conglomerato caotico di azioni extralinguistiche da verbalizzare il parlante, prima di tutto, fa uso di un modulo concettuale acquisito dalla tradizione comunicativa: individuando nella "materia grezza" alcune azioni (o momenti/periodi temporali) secondo lui particolarmente pertinenti, assegna loro lo status di azione centrale (pianeta), per poi distribuire loro intorno le rimanenti azioni in funzione di satelliti. Questo principio ordinativo - centro vs. periferia, o meglio centro, periferia immediata e periferia mediata - può venire poi impresso ricorsivamente su insiemi più o meno ampi di azioni. Alla costellazione concettuale, ${ }^{2}$ sul versante espressivo, corrispondono, in italiano, due principali set di paradigmi verbali. Il graf. 1 illustra le corrispondenze tra i ruoli/le funzioni nella costellazione concettuale: centro, periferia immediata (posteriorià, s imultaneità, anteriorità rispetto al centro) e periferia mediata (varie anteriorità, simultaneità e posteriorità ${ }^{3}$ rispetto ai punti a loro volta anteriori al

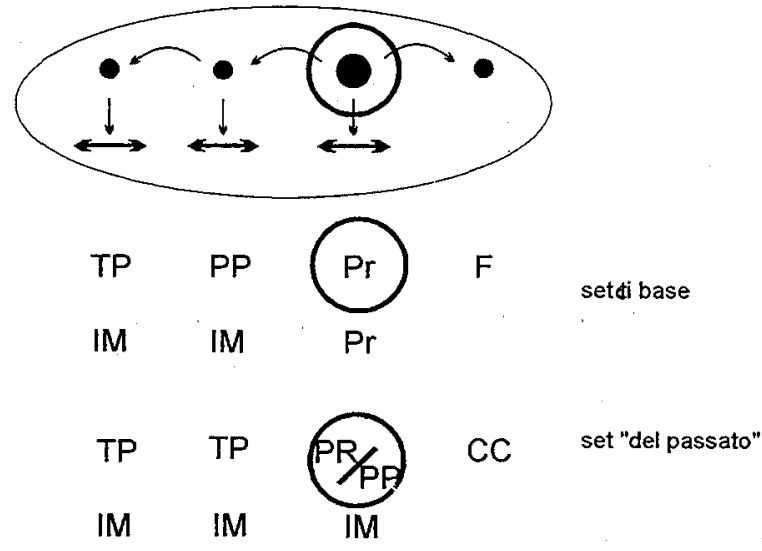

graf. 1

1 Sono consapevole dell'esistenza di parametri di carattere modale, alcuni di loro studiati e presentati in modo esemplare da Bazzanela (1990), da Bertinetto (1986b), da Korzen (2002) e da altri, mi preme però approfondire soprattutto l'indagine del versante tempo-aspettuale - anche nei casi in cui l'argomento riguarda il modo congiuntivo (cfr. Miklič (1991a) e (1991b)).

2 È rappresentata qui solo per mezzo delle più frequenti relazioni di temporalità relativa, senza possibili modificazioni modali.

3 Azioni posteriori rispetto a punti anteriori, per non appesantire ulteriormente il disegno, sono state omesse. 
centro) da una parte, e, dall'altra, i paradigmi verbali componenti i due raggruppamenti maggiormente impiegati: set di base (con il Presente (Pr) al centro) e set "del passato" (con, al centro, generalmente un passato remoto (PR) o un passato prossimo (PP)). Mentre quest'ultimo è specializzato per le situazioni nel passato, il set di base, che può pure servire al riferimento al passato, si usa per tutte le rimanenti sfere temporali. Per il passato disponiamo pertanto di più moduli espositivi: ho chiamato (a) procedimento fondamentale l'esposizione tramite il set "del passato"; (b.) procedimento storico l'esposizione tramite il set di base; e infine (c.) procedimento combinato l'esposizione tramite la combinazione del Presente storico (PrSt) (per il centro) con i paradigmi del set "del passato" (per la periferia). Ecco qualche illustrazion, ${ }^{4} \mathrm{e}$ con azioni centrali designate da maiuscole:

\section{Procedimento fondamentale}

(8)

Ferdinand de Saussure NACOUE a Ginevra il 26 novembre 1857. La famiglia era tra le più famose della città: il capostipite era stato un Mongin (...) (DeM 285)

(PR, IM, TP)

\section{Procedimento storico}

(9)

Ormai il Mémoire è apparso da un anno e, ad onta d'ogni ostilità, il nome di S. è ben noto: poco prima di laurearsi, il giovane SI PRESENTA alle esercitazioni di un valente germanista (...) (DeM 294) (PPSt, PrSt, PrSt)

\section{Procedimento combinato}

(10)

S. EVITA, con sicuro giudizio, il glottologo cattedratico e FREQUENTA invece le lezioni di grammatica greca e latina d'un privat-docent, Louis Morel, che ripeteva, in pratica, quel che l'anno prima aveva appreso a Lipsia nelle esercitazioni e nei seminari di Georg Curtius. (DeM 291) (PrSt, IM, TP)

\section{Ruoli concettuali e punto di riferimento}

La batteria (1)-(7) mostra con chiarezza che i TP e gli IM non servono solo per il riferimento ad azioni nel passato del parlante. E infatti, come illustrato dal graf. 2, la costellazione concettuale di relazioni tempo-aspettuali (con appositi mezzi espressivi) riguarda tutte le sfere temporali. Partirò dalla situazione nel passato (al procedimento storico) - più copiosamente rappresentata - per poi passare a quelle nell'attualità, nell'attualità allargata, nell'avvenire e nell'extratemporalità.

4 Si tratta di segmenti testuali di T. De Mauro, "Notizie biografiche e critiche su F. de Saussure" in de Saussure (1983). 


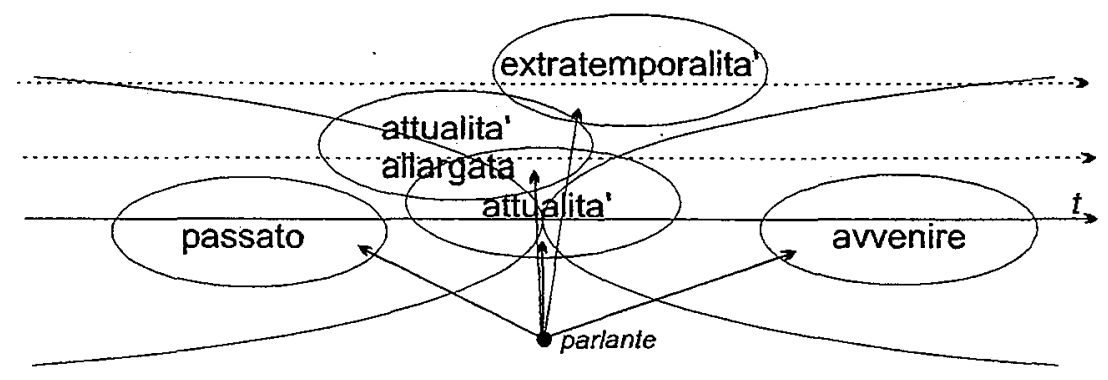

graf. 2

Passiamo quindi all'argomento centrale: occorrenze apparentemente non motivate di TP e di IM. Ora, secondo la teoria che adopero, il valore essenziale del Trapassato e dell'Imperfetto nel set di base ${ }^{5}$, come illustrato nel graf. 3, sarebbe:

TP: azione anteriore rispetto a un "punto" a sua volta anteriore al centro IM: azione simultanea rispetto a un "punto" a sua volta anteriore al centro



graf. 3

Appare subito evidente il ruolo cruciale del punto/momento/periodo di riferimento, dell'elemento di mediazione cioè, tra il centro (al Pr, se esplicitato) e l'azione denotata dal TP o dall'IM. Spesso i ruoli previsti nella costellazione sono effettivamente ricoperti (linguisticamente esplicitati), come nell'es. (11), un riassunto di azioni immaginate nel passato: l'azione focale (PrSt: si incontrano), la prima anteriorità (PPSt: è morto), che costituisce al contempo il punto di riferimento per le due azioni della periferia mediata, la simultaneità (IMSt: si recava) e l'anteriorità (TPSt: aveva scritto) ${ }^{6}$ :

(11)

Nell'ufficio dell'organizzazione si incontrano Isabel, rimasta senza posto di lavoro, e Balboa. Il nipote di questi, Mauricio, è morto in una sciagura navale mentre SI RECAVA dal

5 Nel set "del passato" i due paradigmi hanno evidentemente anche altre funzioni tempo-aspettuali. Cfr. il graf. 1.

6 A quest'ultima è legato un altro satellite contemporaneo (soffriva). 
Canada alla casa dei nonni. Per consolare la nonna che soffriva della vita scapestrata di Mauricio, Balboa le AVEVA SCRITTO delle lettere fingendosi il $\mathrm{n}$ ipote e dandone un ritratto d'uomo pentito e felicemente sposato. Ora si tratta di trovare una coppia disposta a recitare, per alcuni giomi davanti alla nonna, il ruolo di Mauricio e della moglie. (BOM 63, Gli alberi muoiono in piedi)

Dove si trova invece il punto di riferimento per l'IM e il TP in (7)? I Passati Prossimi ha strappato e ha adottato ovviamente non possono candidarsi.

(7)

(...) g li si concede spontaneamente per virtù d'amore (I.35). L a bellezza a cui Amadigi $\underline{\text { SOSPIRAVA e per la quale AVEVA tanto COMBATTUTO e tanto SOFFERTO, gli è data }}$ in dono (...)

In casi come questo, illustrato nel graf. 4, l'azione centrale (al PrSt) (qui si concede) provoca un cambiamento cruciale: compiuta l'azione, è stata creata una nuova situazione rispetto alla quale l'azione stessa è in un certo senso già anteriore. E soprattutto è anteriore la sua parte iniziale, che costituisce il punto di riferimento sia per l'azione vista corsivamente (qui sospirava) sia per quelle anteriori viste globalmente (aveva combattuto e sofferto). Il compimento dell'azione "stacca" ogni collegamento diretto tra la situazione risultante e le azioni valide ancora poco prima, rendendo inaccettabili le soluzioni al Pr e al PP (qui sospira e ha combattuto e sofferto). Come vedremo in seguito, la semantica specifica delle azioni implicate (il punto di riferimento spesso rappresentato da: morte, scoperta, perdita, ottenimento, ritrovamento ecc.) detta, o almeno suggerisce, la scelta del costrutto.

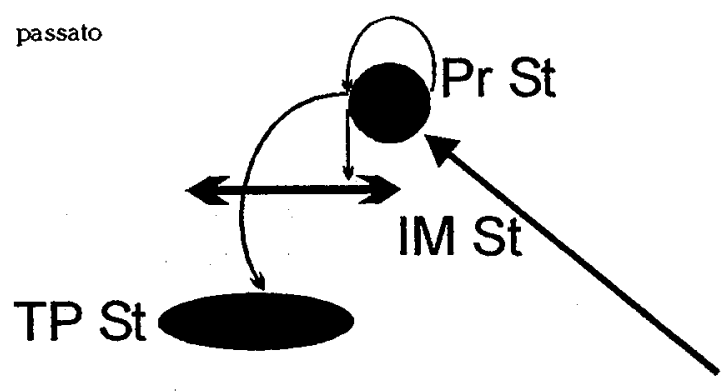

graf. 4

\subsection{Situazione nel passato (al procedimento storico)}

La messe di casi in questione è stata ovviamente molto più abbondante per il passato, ambito in generale maggiormente verbalizzato, che per le altre sfere temporali. Per mancanza di spazio concesso propongo solo pochissimi esempi: 
(12)

Mentre l'Alamanni è in carcere, Pompeo Barbetta si appropria una forte somma che quegli gli AVEVA AFFIDATA e, per timore di essere scoperto, soffoca col guanciale la moglie ammalata, la quale nel delirio MINACCIAVA di rivelarne la colpa. (BOM 453, I Barbarò)

A contatto di questa umanità così complessa e primitiva J. raggiunge, di colpo, quella maturità e comprensione della vita di cui egli ERA sempre ANDATO in cerca. (BOM 482-3, Per chi suona la campana)

(...) e sposa un giovane contadino, onesto e buono, che prima AVEVA RIFIUTATO.

(BOM 497, Bianchina)

(...) attraverso la descrizione delle vicende di un uomo di cultura che si rifiuta di accettare la realtà e cerca di subordinarla alla propria concezione del mondo, finendo così per soccombere a un sistema che AVEVA SOTTOVALUTATO. (BOM 464, I bastardi)

La signora Ersilia veglia il marito morto, e, preda d'una follia lucida e tranquilla, intesse con lui un dialogo per potersi finalmente spiegare dopo che per tutta la loro vita non AVEVANO SAPUTO o VOLUTO intendersi. (BOM 208, Ponentina)

Dick si innamora di lei: comincia a bere troppo e a perdere la disinvolta sicurezza che lo DISTINGUEVA. (GAR 878)

(...) non ottiene nulla da colei che SEMBRAVA promettere tutto. (BOM 236, Aperto la notte)

Qualche volta, nella linearizzazione, l'autore dopo il centro (al PrSt) riparte con un sorprendente TP per poi svelare con nuove informazioni (tramite un PPSt, gerundio composto, participio, aggettivo ecc.) che si trattava di seconda periferia, ad es.

Giunge Oreste (...) a cui Menelao AVEVA un tempo PROMESSO la figlia, venendo poi meno alla promessa per darla a Neottolemo. (BOM 174, Andromaca)

Ma Cremete, che in un primo tempo SI ERA DISGUSTATO per lo scandalo, persuaso da Simone, acconsente di nuovo al matrimonio che viene pubblicamente annunziato. (BOM 172, Andria)

Si può avere inoltre una vera e propria anticipazione dell'azione cruciale come in caso di "preludio" (cfr. Miklič (1998)). Con il TP in tali contesti è preannunciato un cambiamento (punto intermedio) e il risultante nuovo stato (il centro): 
(21)

Ma a un tratto la fortuna che gli ERA STATA sempre favorevole gli volta le spalle: la superba casa nuova, (...), ì distrutta completamente da un incendio (...). (BOM 321, L'ascesa di Silas Lapham)

L'IM e il TP appaiono quindi quando l'azione centrale annulla la persistenza dell'azione vista corsivamente o stacca il contatto con l'azione anteriore:

(22)

Ma la madre di Edoardo si oppone a quelle nozze e dà al figlio minore, Roberto, la proprietà che SPETTAVA a Edoardo. (BOM 560, Buonsenso e sensibilità)

(23)

Agamene la consola e la ospita nella sua casa; così la bellissima Melissa diviene l'amante e la modella che lo scultore CERCAVA. (BOM 176, L'anello di Policrate)

(...) lentamente la sua vera natura riaffiora dall'annoso letargo in cui SI ERALASCIATO impantanare; (BOM 507, La bilancia alterata)

Se invece non possiede la forza annullatrice si trovano il PrSt e il PPSt:

(25)

Lucia, corteggiata da Roberto, non esita allora a lasciare l'antico fidanzato che non può più offrirle l'esistenza agiata che ella DESIDERA. (BOM 560, Buonsenso e sensibilità)

(26)

Dopo alcune avventure ritrova Giosiana, che nel frattempo È STATA data forzatamente in isposa a Yvorin, (...) (BOM 561, Buovo d'Antona)

Accanto alla stragrande maggioranza di soluzioni con l'IM:

(27)

(...) che muore tra le fiamme insieme a Creonte che CERCAVA di salvarla. (GAR 855)

(28)

(...) e la salva da uno dei cristiani che STAVA per ucciderla. (GAR 843)

si trovano anche poche eccezioni che non seguono il principio prototipico, spesso prodotti da autori di madrelingua non italiana, ad es.:

(29)

Ma Gian Galeazzo cerca di sopprimere lo zio, e per mezzo d'un segreto manipolatore di veleni uccide il vecchio Barnabò che invano il fedele Enzel CERCA di salvare. (BOM 173, Il castello di Trezzo)

(30)

Al momento di salpare da Livomo per Boston col suo mercantile, David salva un uomo che STA per annegare, Shiloh: (BOM 182, L'angelo orfano) (Lia Wainstein) 
(31)

A poco á poco egli distrugge tutte le certezze di cui VIVE l'innocente e inesperto Kòlja, e che costituiscono il fondo di misteriosa e profonda bellezza dell'anima di ogni fanciullo. Poi gli insinua il desiderio di morire, con una specie di ipnotismo satanico, che emana dalla sua bieca persona. Tutto incomincia a sbiadire agli occhi di Kòlja, il suo cuore si distacca da tutto quello che gli ي̀ caro. (BOM 567, Più dolce del veleno) (Olga Signorelli Resnevich)

\subsection{Situazione nell'attualità}

Si tratta qui di quelle costellazioni di azioni che hanno il fuoco nella situazione comprendente il momento dell'enunciazione, da cui poi vengono calcolate varie anteriorità ( $\mathrm{e}$ anche posteriorità, che però qui non ci interessano). Anche se le azioni anteriori sono in realtà già nel passato del parlante, dal momento che il centro è nell'attualità, le varie periferie saranno considerate appartenenti a questa sfera temporale.

Spesso le varie profondità sono esplicitate linguisticamente, come nel seguente esempio, che presenta sia la prima anteriorità (PP) che quella seconda (TP):

- Ho rotto il vaso che ci AVEVA REGALATO la nonna! (LSE 3320-42)

Altre volte, l'azione di prima anteriorità, che costituisce al contempo il punto di riferimento per 1 'azione al $\mathrm{TP}$, è ricavabile $\mathrm{d}$ al contesto s ituazionale. $\mathrm{N}$ egli e sempi introduttivi (1.a-1.e) potrebbe essere individuato forse in: eccoci arrivati (1.a); si è rotto! (1.b e 1.c); hai detto bene (1.d) e infine è già tornato!? (1.e). Similmente in casi come il seguente (cfr. il graf. 5):

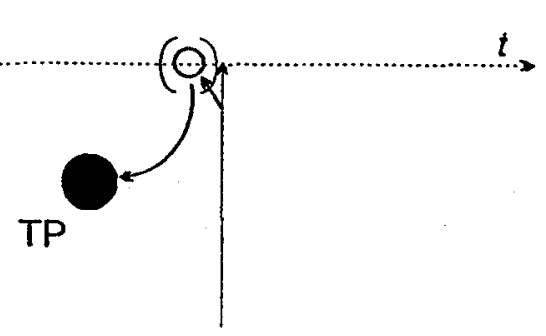

graf. 5

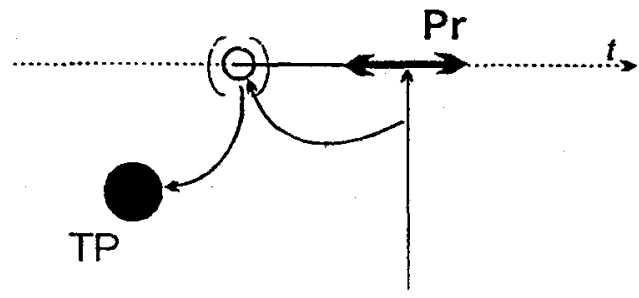

graf. 6

(33)

In una sala da gioco, al tavolo della roulette.

- Giocate il numero corrispondente alla vostra età - consiglia un tale ad una bella signora.

- Ah, bene! - dice lei, e gioca il 26.

Esce il 36.

- Ma ve l'AVEVO DETTO! - commenta lui. (LSE 2775-10)

(azioni in successione: lui consiglia, lei sceglie, lui commenta) 
Sono interessanti alcune combinazioni di un TP con un Pr che si riferisce a uno stato nell'attualità vera e propria. Il Trapassato testimonia la presenza di un evento intermedio - spesso l'inizio dello stato (cfr. il graf. 6) - nella coscienza del parlante:

Carlo, ho quell'asse di legno per lo steccato che mi AVEVI CHIESTO. (LSE 3060-25) (ad es. l'ho trovato)

[...] quando sto lavorando, lavoro concretamente, quando esco di qui stacco di netto. La cosa brutta sa cos'è (me lo AVEVANO DETTO e lo sto verificando sulla mia pelle)? (Gin 252) (è infatti successo)

(un commerciante viaggiatore uscendo dall'ufficio di una Agenzia matrimoniale con una donna al braccio)

- Se penso che ERO ENTRATO soltanto per offrire un aspirapolvere... (LSE 3130-18) (ho trovato moglie)

(37)

(la moglie rientrando in casa al marito)

- Su caro: AVEVI PROMESSO che se io avessi verniciato la parte inferiore delle finestre, tu avresti verniciato quelle in alto! (LSE 3257-23) (ho fatto la mia parte)

Anche l'uso dell'Imperfetto è legato all'ancoraggio di una situazione a un punto di riferimento anteriore alla situazione valevole al momento dell'enunciazione, che pure va dedotto dal contesto globale. Spesso, ma non sempre, si trova nell'atto linguistico precedente:

(38)

Un ometto dall'aria assorta, corre lungo una strada provinciale. Indossa maglia e calzoncini da ciclista ed ha una gomma da bicicletta incrociata sul petto.

- Ma che cosa fate? - gli chiede un contadino.

- Il Giro d'Italia!

- A piedi?

- Oh, grazie! - esclama il corridore illuminandosi in volto. - NON RIUSCIVO assolutamente a ricordarmi che cosa avevo dimenticato alla partenza! (LSE 2877)

(il principale detta alla segretaria)

- Io sono onorato...

- Oh! - esclama con un gridolino la ragazza. - ERO convinta che vi chiamaste Alberto! (LSE 2769-10)

(40)

Lei: Non dormi?

Lui: E tu?

Lei: STAVO PENSANDO ... (...) (RAI 1, pubblicità per la Panda) 
(41)

(la moglie al marito sdraiato per terra)

- Ma allora quel filo PORTAVA corrente... (LSE 3163-42)

(42)

(Tilli, parlando con un'amica nel proprio appartamento sta cercando da un po' la borsetta. Alla fine, camminando verso l'oggetto) - L'ho trovata. Ecco dove ERA! (L'incantesimo, RAI 1, 6.11. '02)

(dopo alcuni minuti di conversazione al telefono, Alberto all'amico che ha chiamato)

"Ma tu... tu CERCAVI Micòl, non è vero?" disse infine lui, come ricordandosi

"Già" risposi. "Ti dispiace passarmela?" (GFC 112)

(44)

(Tilli, da cui temporaneamente abita Martina, apre ad Andrea)

Andrea: "Ciao, Tilli. Scusa l'ora..."

Tilli: "Ciao, Andrea. Tu CERCAVI Martina, vero? Non c'è, mi dispiace.

(L'incantesimo, RAI 3, 6.11. '02)

(45)

(il vigile al conducente nella macchina)

- Si rilassi: non ha commesso nessuna infrazione. Ė che sto qui da ore e VOLEVO fare quattro chiacchiere. (LSE 3637-15) (L'ho fermata perché...)

Seccato per aver atteso oltre un quarto d'ora prima che venissero a prendere la sua ordinazione, un tale dice al cameriere:

- VOLEVO ordinare delle lumache, ma se per caso è proprio lei che va a prenderle, temo che le sfuggiranno, perché sono troppo veloci. (LSE 3682-10) (ho capito)

(47)

Che cosa fai stasera?

Beh, ANDAVO al cinema. (intenzione al momento della domanda)

Questo ancoraggio a un momento diverso dal momento dell'enunciato può essere sfruttato per alcune sfumature modali. Cfr. in particolare Bazzanella (1990).

\subsection{Situazione nell'attualità allargata}

Questa sfera temporale spesso non è temporalmente molto articolata e oltre all'azione centrale, come in Negli ultimi tempi mi sveglio alle sei di mattina, appare solo occasionalmente qualche satellite immediato, ad es. di anteriorità:

(due mogli al bar)

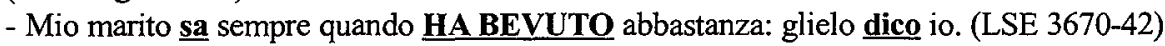

Eppure si trova anche qualche esempio di satellite mediato (simultaneità nell'anteriorità), come in questo esempio, in cui il punto di riferimento anteriore al centro (o 
meglio i punti di riferimento) è reso da un avverbiale (nella precedente discussione) (cfr. il graf. 7):

(49)

A Tuscaloosa (Stati Uniti), la giovane signora Mary Pellman ha chiesto il divorzio perché il marito la batte spesso. Inoltre, come ella ha voluto puntualizzare davanti al giudice, "il mascalzone non ha neppure la cortesia di scusarsi" quando lei gli dimostra che nella precedente discussione lui AVEVA torto marcio. (LSE 2994-4)

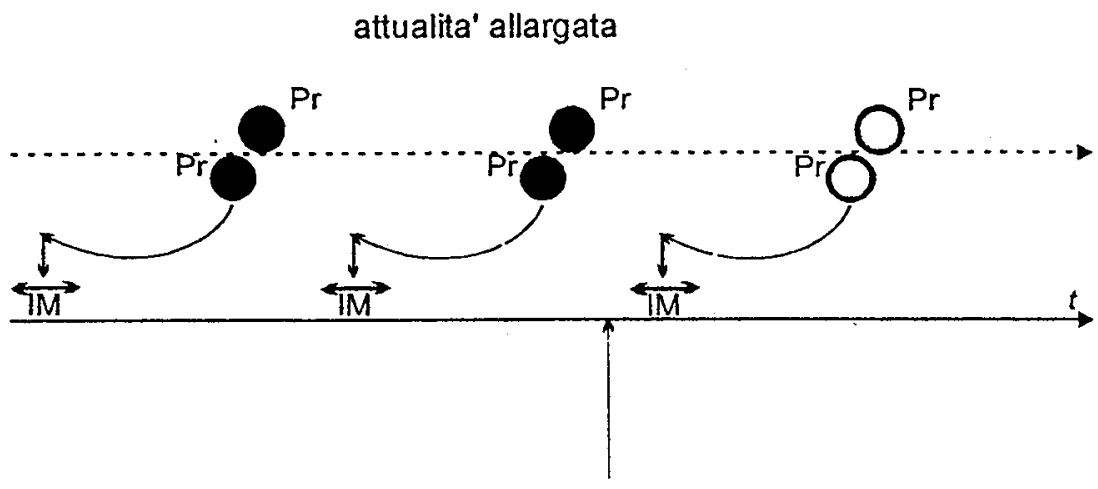

graf. 7

\subsection{Situazione nell'avvenire}

Sono ampiamente conosciuti gli usi del Passato Prossimo per azioni nell'avvenire, possibili però solo se l'azione anteriore è vista globalmente (v. il graf. 8, per (51)):

(50)

- Portami il giomale quando HAI FINITO di leggerlo!

(il medico al paziente)

- Voglio che smetta di fumare e che perda dieci chili! Poi torni da me, e mi dica come $\underline{\text { HA }}$ FATTO. (LSE 3628-21)

Se, invece, il tipo d'azione e la situazione esigono l'ottica corsiva, appare l'IM, come nei seguenti enunciati (v. il graf. 9, per il primo) ${ }^{7}$ :

(in sala operatoria, un chirurgo all'altro)

- E va bene, faccia come le pare, ma l'autopsia proverà che AVEVO ragione io!

(LSE 3571-15)

7 È chiaro che se l'azione è vista come attuale nel momento dell'enunciazione viene invece espressa dal Pr:

(52a)

(un bambino all'altro)

- Adesso verrà la mamma: dirà lei chi di noi due HA ragione. (LSE 3568-37) 


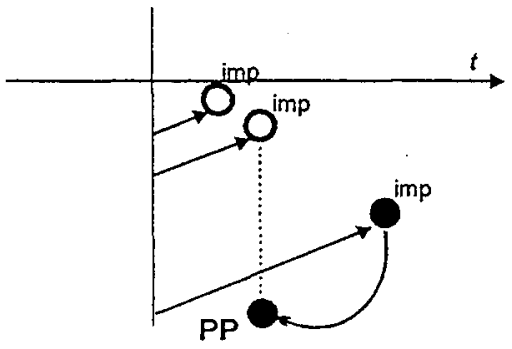

graf. 8

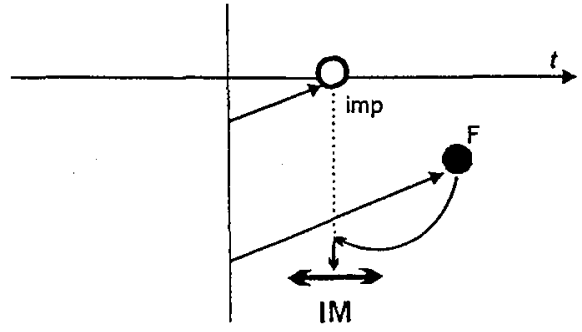

graf. 9

(53)

(riferendosi a Napoleone nella sua tipica posa, un ufficiale militare all'altro)

- È un trucco: dice che quando la gente avrà scordato le sue battaglie, continuerà sempre a chiedersi perché TENEVA la mano infilata lì... (LSE 3550-43)

(il Reggente al piccolo Principe ereditario)

- Ricorda di comportarti sempre bene! Un giorno sarai re: la gente mi chiederà com'ERI da ragazzo, e glielo racconterò! (LSE 3669-42)

\subsection{Situazione nell'extratemporalità}

Anche nel caso di extratemporalità ci interesseranno azioni nell'anteriorità rispetto al centro, che se si tratta di azioni considerate globalmente e legate direttamente al centro vengono rese regolarmente dal Passato Prossimo ${ }^{8}$ :

(55)

In amore, come in tutte le cose, l'esperienza è un medico che arriva quando la malattia $\underline{\mathbf{E}}$ FINITA. (LSE 2775-48)

Eppure, quando necessario, appaiono anche il TP e l'IM. Così ad es. troviamo ricoperti i posti sia della prima anteriorità (PP: ha scritto) che della seconda (TP: si era ripromesso):

(56)

James Barrie, l'autore di Peter Pan, disse una volta: La vita è un quaderno sul quale ciascuno di noi ha intenzione di scrivere una storia, e finisce invece con lo scriverne un'altra. E nessuna umiliazione è peggiore di quella che l'uomo prova nel confrontare ciò che ha scritto con quello che SI ERA RIPROMESSO di scrivere. (LSE 2850-12)

8 Il Passato Remoto è invece estremamente raro:

(55a)

Non si compiange mai chi ANDÒ cercando guai. (LSE 2775-48) 
Nei seguenti enunciati, e anche in (4), il punto di riferimento per l'azione anteriore può nascondersi in un'azione al $\operatorname{Pr}$ (svaniscono, muore), a sua volta anteriore al centro (sembrano, vengono bruciate) (v. il graf. 10)

Il guaio delle buone occasioni è che quando svaniscono sembrano migliori di quando $\underline{\text { SI }}$ ERANO PRESENTATE. (LSE 3577-48)

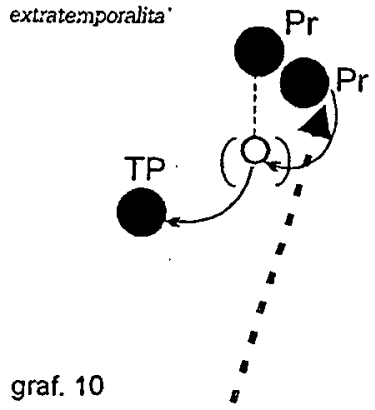

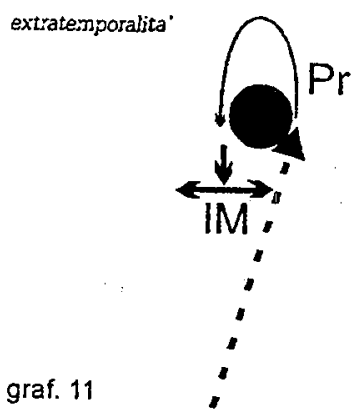

oppure, come abbiamo visto in (7) e in tanti altri casi, è costituito dalla stessa azione centrale al Pr, che "stacca" la situazione risultante dalla periferia (cfr. il graf. 4):

(58)

Gli zingari seguono tuttora le precise regole della tradizione. Quando muore uno di loro, ad esempio, dopo la sepoltura il letto, gli indumenti e gli oggetti che gli ERANO APPARTENUTI vengono bruciati. Le stoviglie $e$, in genere, tutte le cose che non possono essere distrutte con il fuoco vengono invece fatte a pezzi. (LSE 3524-12)

(59)

"Questa, dice il protagonista, è la storia più triste che abbia mai sentito". Essa viene narrata senza un piano prestabilito, con il procedimento tortuoso di chi, a poco a poco, vede farsi luce su un passato che AVEVA del tutto FRAINTESO. (Il buon soldato, BOM 560)

Nella sua plasticità essa corrisponde all'aspirazione estetica e, coerentemente, il desiderio si fissa nell'amplesso dell'acqua, che cinge la pietra. Con questa visione cristallina si chiude, come S'ERA APERTO, il poemetto. (BOM 124, L'Ambra)

Similmente vale per l'IM. Mentre nell'es. successivo il punto di riferimento per l'azione simultanea è esplicitato da un avverbiale (l'anno prima) (cfr. il graf. 7):

(61)

(due uccelli osservando dagli scogli la gente sulla spiaggia sottostante)

- È davvero inspiegabile come ogni anno riescano a trovare lo stesso posto in cui ERANO l'anno prima. (LSE 3671-8) 
altre volte il compimento dell'azione centrale spinge il punto di riferimento per l'azione simultanea indietro, al momento del suo inizio (v. il graf. 11):

Accadono in un punto cose strane, che PAREVANO impossibili o lontane. (DP 70)

D'altra parte se la sceneggiatura è spesso elemento determinante per ricostruire la coerenza testuale, si possono dare casi in cui un testo modifica e trasforma completamente la sceneggiatura di riferimento a cui ERAVAMO abituati. (Vio 31)

\subsection{Altri casi}

A questo punto possiamo affrontare la situazione in (6a): perché dopo un PR, per un'azione successiva viene usato il TP? Va subito detto che il fenomeno non è limitato al TP: nello stesso modo vengono adoperati altri paradigmi atti a segnalare l'anteriorità rispetto al punto di riferimento. Quest'ultimo, però, qui non sta nell'azione indicata dal paradigma precedente (in 6a: si gettarono all'attacco) bensì in un momento posteriore segnalato da un avverbiale (in 6a: in un batter d'occhio). Ecco casi analoghi con altri paradigmi composti: Futuro Composto (Passato Prossimo) e Passato Prossimo storico:

(64)

Un momento ancora e AVRÒ FINITO (ho finito).

Il matrimonio fallisce. Pavlína riprende i suoi rapporti con l'amante di prima. La madre amministra la bottega di emla e in breve tempo l'HA già INGHIOTTITA. (BOM 87, Alla bottega inghiottita)

Ma la fortuna e la sua presenza di spirito gli vengono in aiuto. Dopo una settimana egli è diventato l'uomo più celebre di Londra, ricchissimo a un tempo di credito e di debiti.

(BOM 506, Il biglietto di un milione di sterline)

In (6a), "Mio zio Ernesto AVEVA COMPRATO un'auto. Alla prima uscita, però, andò a sbattere contro un albero.(...)", abbiamo la tipica situazione di preludio (cfr. Miklič (1998b)). La forma composta (vale lo stesso per il Passato Prossimo usato in combinazione con il Presente), tipica della periferia, preannuncia l'azione cruciale: le azioni in successione sono così distribuite per importanza in centro vs. periferia. È interessante che nell'inchiesta svolta sulla distribuzione del Perfetto Semplice o Perfetto Composto (Passato R emoto o P assato Prossimo) (Bertinetto/ S quartini (1996: 407)) gli autori hanno constatato che in una situazione analoga (ibid. p. 412: Non ho più la macchina, COMPRAR-LA due anni fa, AVERE subito un incidente e VENDER$L A$ poco dopo) alcuni degli intervistati, ai quali si chiedeva di sostituire gli infiniti con uno dei Perfetti, per il primo verbo (comprar-la) avevano scelto invece un Trapassato. 
In (5) le autrici si riferiscono al discorso interrotto (periferia mediata) per affrontare un altro argomento (periferia immediata).

\section{Conclusione}

Mediante la discussione di una serie di occorrenze, nell'ambito del set di base, di TP o di IM apparentemente "indipendenti" o non legati, usati cioè da soli o con un Presente, ho cercato di mostrare 1. che si tratta solo di variazioni contratte della costellazione prototipica con il centro al $\operatorname{Pr}$ e con l'indispensabile punto di riferimento, in casi standard espresso da un PP, ma qui non esplicitato, e quindi ancora da individuare nel co- o contesto; 2 . che 1'approccio teorico adoperato, che postula costellazioni di azioni in rapporti tempo-aspettuali complessi in tutte le sfere temporali, prevede automaticamente anche casi di IM e TP per azioni nell'avvenire, extratemporalità, attualità allargata e attualità (in senso largo, evidentemente, con varie anteriorità e posteriorità) e si presta bene alla discussione della problematica del funzionamento tempoaspettuale dei paradigmi verbali italiani. Rappresenta, ovviamente, solo una componente ed è sufficiente per l'analisi di un certo tipo di problemi. All'occorrenza, va pertanto completato da altri parametri di tipo sintattico, lessicale, pragmatico ecc.

\section{Corpus}

(Ber) Bertinetto, P. M. (1986) Tempo, aspetto e azione nel verbo italiano. Il sistema dell'indicativo. Firenze:

Accademia della Crusca.

(BOM) Dizionario Bompiani delle Opere e dei Personaggi. Milano: Bompiani, 1983.

(CD) Thornton, A. M./ M. Voghera (1985): Come dire (storia, grammatiche e usi della lingua italiana). Bergamo: Minerva Italica.

(DeM) De Mauro, T. (1983) Notizie biografiche e critiche su F. de Saussure. In: Ferdinand de Saussure, Corso di linguistica generale. Roma/Bari: Laterza, 285-355.

(DP) Selene, A. (1990) Dizionario dei proverbi. Milano: Orsa Maggiore.

(Eco) Eco, U. $\left({ }^{23} 1988\right)$ Il nome della rosa. Milano: Bompiani.

(GAR) Enciclopedia Garzanti della letteratura. Milano: Garzanti, 1979.

(GFC) Bassani, G. (1991) Il giardino dei Finzi-Contini. Milano: Arnoldo Mondadori.

(Gin) Ginsborg, P. (cur.) (1994) Stato dell'Italia. Il Saggiatore. Milano: Bruno Mondadori.

(Kor) Korzen, I. (2002) Il Trapassato Prossimo in un'ottica pragmatico-testuale. In: H. Jansen et al. (cur.), L'infinito \& oltre. Omaggio a Gunver Skytte. Odense University Press: Odense, 203-226.

(LSE) La Settimana Enigmistica

(RAI) Telenovela L'incantesimo della RAI 1

(Vio) Violi, P./G. Manetti (1979) L'analisi del discorso. Milano: Espresso Strumenti.

\section{Bibliografia}

BAzZANELla, C. (1990) Modal uses of the Italian indicativo imperfetto in a pragmatic perspective. Journal of Pragmatics 14 (3), 439-457.

Bazzanella, C. (1994) Le facce del parlalre. Un approccio pragmatico all'italiano parlato. Firenze/Roma: La Nova Italia.

Bazzanella, C. (2000) Tense and Meaning. In: D. Marconi (cur.), Knowledge and Meaning - Topics in Analytic Philosophy. Vercelli: Mercurio, 177-194.

BÄUERLE, R. (1979) Temporale Deixis, temporale Frage. Tübingen: Niemeyer. 
Bertinetro, P. M. (1986a) Intrinsic and Extrinsic Temporal References. On Restricting the Notion of 'Reference Time': In: V. Lo Cascio/C. Vet (cur), 41-78.

Bertinetto, P. M. (1986b) Tempo, aspetto e azione nel verbo italiano. Il sistema dell'indicativo. Firenze: Accademia della Crusca.

Bertinetto, P. M. (1991) Il verbo. In: L. Renzi/G. S alvi (cur.), Grande grammatica di consultazione. Vol. II. Bologna: Il Mulino, 113-161.

Bertinetto,P. M. (1997) Il dominio tempo-aspettuale. Demarcazioni, intersezioni, contrasti. Torino: Rosenberg \& Sellier.

Bertinetto, P. M. (1999) Sperimentazioni linguistiche nella narrativa del Novecento: variazioni sul Tempo Verbale 'propulsivo'. Atti dell'Accademia Lucchese di Scienze, Lettere ed Arti. Seconda serie, tomo XXVIII, 41-102.

Bertinetto, P. M./M. SQuartini (1996) La distribuzione del Perfetto Semplice e del Perfetto Composto nelle diverse varietà di italiano. Romance Philology XLIX/4, 384-419.

BinNICK, R. (1991) Tense and the Verb. (A Guide to Tense and Aspect.) New York/Oxford: Oxford University Press.

BronZWAER, W. J. M. (1970) Tense in the Novel. (An Investigation of Some Potentialities of Linguistic Criticism.) Groningen: Wolters-Noordhoff.

Comrie, B. (1985) Tense. Cambridge: Cambridge University Press.

COOPER, R. (1986) Tense and Discourse Location in Situation Semantics. Linguistics and Philosophy 9, 17-36.

DECLERCK, R. (1991) Tense in English. Its structure and its use in discourse. London/New York: Routledge.

DINSMORE, J. (1981) Tense choice and time specification in English. Linguistics 19; 475-494.

DRY, H. (1981) Sentence aspect and the movement of narrative time. Text 1 (3), 233-240.

Engel, D. M. (1990) Tense and Text. (A Study of French Past Tenses.) London and New York: Routledge.

FLeIsCHMAN; S. (1985) Discourse functions of tense-aspect oppositions in narrative: toward a theory of grounding. Linguistics 23, 851-882.

Fleischman, S. (1990) Tense and Narrativity. London: Routledge.

FLUDERNIK, M. (1993) The Fictions of Language and the Languages of Fiction. The Linguistic Representation of Speech and Consciousness. London: Routledge.

GENETTE, G. (1994) Die Erzählung. München: Wilhelm Fink.

Korzen, I. (2002): Il Trapassato Prossimo in un'ottica pragmatico-testuale. In: H. Jansen et al. (cur.), L'infinito \& oltre. Omaggio a Gunver Skytte. Odense University Press: Odense, 203-226.

LÄMMERT, E. (1972) Bauformen des Erzählens. Stuttgart: Metzler.

Lo CAScio, V. (1984) Deissi e anafora nel testo: alla ricerca di un punto di riferimento. In: L. Coveri (cur.), Linguistica testuale, Roma: Bulzoni, 207-236.

Lo CASCIO, V./C. VET (1986) Temporal Structure in Sentence and Discourse. Dordrecht: Foris.

MIKLIČ, T. (1983) L'opposizione italiana PERFETTO vs IMPERFETTO e l'opposizione slovena DOVRŠNOST vs NEDOVRŠNOST nella verbalizzazione delle azioni passate. Linguistica XXIII, 53-123.

Miklic, T. (1986) Strutturazione del testo tramite forme verbali (analisi contrastiva). Scuola Nostra 17-18, 119-164.

Miklic, T. (1991a) Forme verbali italiane: come vengono presentate dalle grammatiche e come funzionano nei testi. Scuola Nostra 23, 87-103.

Miklic, T. (1991b) La forma verbale e la sua funzione nel testo: servigi testuali del trapassato del congiuntivo. In: L. Giannelli et al. (cur.), Tra Rinascimento e strutture a ttuali. Saggi dilinguistica italiana. I. Torino: Rosenberg \& Sellier, 319-330.

Miklic, T. (1991c) Presenza e valori del passato remoto in riassunti di opere letterarie. Linguistica XXXI, 249-258.

Miklic, T. (1997) Segnalazione della temporalità nel testo: che cosa aiuta il ricevente a collocare le azioni sull'asse temporale. In: L. Agostiniani et al. (cur.), Atti del Terzo Convegno della Società Internazionale di Linguistica e Filologia Italiana. Napoli: Edizioni Scientifiche Italiane, 477-505.

Miklic, T. (1998a) La distribuzione dei paradigmi verbali nei compendi di opere letterarie (italiano e tedesco a confronto.) In: P. Cordin et al. (cur.), Parallela 6: Italiano e tedesco in contatto e a confronto. Trento: Università degli Studi, 451-467.

Miklic, T. (1998b) Uso cataforico del trapassato prossimo italiano: un espediente testuale per la messa in rilievo. Linguistica XXXVIII/2, 183-195.

Miklic, T. (ics) Interpretazione della funzione testuale dei paradigmi verbali italiani: tentativo di un modello d'analisi integrato.

Miklic, T. (ics) Testi narrativi, azioni centrali e paradigmi verbali italiani.

NerbonNe, J. (1986) Reference time and time in narration. Linguistics and Philosophy 9, 83-95.

RiCoeur, P. (1980) Narrative Time. Critical Inquiry, Autumn 1980, 169-190. 
SCHIFFRIN, D. (1981) Tense variation in narrative. Language 57, 45-62.

SCHOPF, A. (1984) Das Verzeitungssystem des Englischen und seine Textfunktion. Tübingen: Niemeyer.

SMITH, C. S. (1980) Temporal structures in discourse. In: C. Rohrer (cur.), Time, Tense and Quantifiers. Tübingen: Niemeyer, 357-374.

Smith, C. S. (1991) The Parameter of Aspect. Dordrecht/Boston/London: Kluwer Academic Publishers.

VENDLER, Z. (1957) Verbs and times. The Philosophical Review LXVI, 143-160.

VIKNER, S. (1985) Reichenbach revisited: one, two, or three temporal relations? Acta linguistica Hafniensia 19.2, $81-98$.

WEINRICH, H. (1964) Tempus: Besprochene und erzählte Welt. Stuttgart: Kohlhammer.

Weinrich, H. (1989) Grammaire textuelle du français. Paris: Les Editions Didier.

WunderLich, D. (1970) Tempus und Zeitreferenz im Deutschen. München: Hueber.

Povzetek

\section{NEKATERE TEMPORALNO-ASPEKTUALNE RABE ITALIJANSKIH GLAGOLSKIH PARA- DIGEM TRAPASSATO PROSSIMO IN IMPERFETTO}

V prispevku sledi avtorica dvema ciljema: v pretresu danega jezikovnega gradiva, osredotočenega na nekatere navidezno samovoljne oz. nepričakovane rabe paradigem TRAPASSATO PROSSIMO in IMPERFETTO, skuša po eni strani ob ponujenih razlagah opozoriti na primernost oz. učinkovitost izbranega teoretičnega pristopa v proučevanju funkcij italijanskih osebnih glagolskih oblik: namreč njihovo razmestitev v dve konstelaciji, dva temeljna seta gl. paradigem, preteklostnega in osnovnega, ki govorcu služita za izražanje dobnostno-aspektualnih relacij med dejanji v katerikoli časovni sferi. Z obravnavanimi zgledi pokaže, da se $\mathrm{v}$ osnovnem setu, ki ima v središču paradigmo PRESENTE in ki je uporabljiv za izražanje dejanj $v$ vseh časovnih sferah, TRAPASSATO PROSSIMO (TP) in IMPERFETTO (IM) lahko nanašata tudi na dejanja v nepreteklosti, in torej ne moreta veljati le za "pretekla časa". Po drugi strani ponuja v podporo raziskovalcem, ki za izbiro TP in IM postulirajo bistveno vlogo referenčne točke, predloge interpretacij za tiste primere, $\mathrm{v}$ katerih ta točka ni neposredno eksplicirana. Avtorica skuša prepričati, da navidezno "presenetljive" rabe logično temeljijo v izhodiščno postuliranih dobnostno-aspektualnih vrednostih obeh obravnavanih paradigem. 